\title{
Rotation Stabilises 2D Skyrmions
}

\section{Rossen Dandoloff}

Department of Condensed Matter Physics and Microelectronics, Faculty of Physics, Sofia University, Sofia, Bulgaria

Email: rdandoloff@yahoo.com

How to cite this paper: Dandoloff, $\mathrm{R}$. (2020) Rotation Stabilises 2D Skyrmions. Journal of Modern Physics, 11, 1326-1329.

https://doi.org/10.4236/jmp.2020.119083

Received: August 12, 2020

Accepted: September 14, 2020

Published: September 17, 2020

Copyright (c) 2020 by author(s) and Scientific Research Publishing Inc.

This work is licensed under the Creative Commons Attribution International License $(\mathrm{CC}$ BY 4.0).

http://creativecommons.org/licenses/by/4.0/

\section{Open Access}

\begin{abstract}
We present a study of 2D Skyrmions under rotation. The purpose of this study is to establish the result of the rotation on the stability of $2 \mathrm{D}$ Skyrmions. Usually the 2D skyrmions are metastable unless the underlying geometry introduces a characteristic length or e.g. magnetic field is present in the problem. We have used a previous study of the rotating plane which proves the appearance of curvature as a result of the rotation. The curvature of the rotating disk introduces length and plays here the role of strength of the angular momentum of the field. We have shown that this additional length scale introduced by the curvature stabilises the 2D Skyrnions under rotation.
\end{abstract}

\section{Keywords}

2D Skyrmions, Stability, Rotations

\section{Introduction}

The 2D skyrmions have played significative role in the modelling of high Tc superconductivity based on the 2D quantum antiferromagnet, the quantum Hall effect etc. One significant feature of the static model as it has been formulated by Belavin and Polyakov [1] is that the skyrmions are metastable. If the field is modelled by normalised unit vector field $\mathbf{n}$ (with $\mathbf{n}^{2}=1$ ) we have that:

$$
H(\lambda)=\iint\left[\left(\frac{\partial \mathbf{n}}{\partial(\lambda x)}\right)^{2}+\left(\frac{\partial \mathbf{n}}{\partial(\lambda y)}\right)^{2}\right] d(\lambda x) d(\lambda y)=H
$$

where $\lambda$ is a scalar. This means that the whole skyrmion may be scaled down to a point. In order to stabilise the skyrmions one has to introduce length in the problem or to take out a hole in the middle of the plane or to apply external magnetic field [2]. To illustrate this we will consider only the example of the nonlinear sigma model on a cylinder which exhibits skyrmions that are stable and whose length is correlated with the radius of the cylinder [3]. 
Let the radius of the cylinder be $\rho_{0}$. In order to incorporate the constraint $\mathbf{n}^{2}=1$, we will work with the two fields $(\theta, \Phi)$ where $\mathbf{n}=$ $(\cos \theta, \sin \theta \cos \boldsymbol{\Phi}, \sin \theta \sin \boldsymbol{\Phi})$. Here $\theta$ is the co-latitude and $\Phi$ is the azimuthal angle. Then, in cylindrical coordinates $(\rho, x, \varphi)$ the Hamiltonian is as follows [3]

$$
\begin{array}{r}
H=J \iint_{\text {cylinder }}\left[\left(\partial_{x} \theta\right)^{2}+\sin ^{2} \theta\left(\partial_{x} \Phi\right)^{2}\right. \\
\left.+\frac{\left(\partial_{\varphi} \theta\right)^{2}}{\rho_{0}^{2}}+\frac{\sin ^{2} \theta}{\rho_{0}^{2}}\left(\partial_{\varphi} \Phi\right)^{2}\right] \rho d x d \varphi
\end{array}
$$

where $J$ is the spin-spin coupling constant. If in this Hamiltonian we replace $x \rightarrow \lambda x$ then $H(\lambda) \neq H$ i.e. the Hamiltonian is no more scale invariant and the skyrmion cannot be shrunk to a point. In what follows we would like to investigate if there are even more methods of stabilising the skyrmions.

\section{The Rotation and Its Cosequences}

Now we will present a novel method for stabilising skyrmions, namely putting the disk (we are considering a disk with finite radius (where skyrmions are metastable too) [4] rather tnan an infinite plane) in rotation. We consider two coordinate systems on the disk, one is fixed $(x, O, y)$ and one which is rotating with the disk: $\left(x^{\prime}, O, y^{\prime}\right)$. The two coordinate systems are related by:

$$
\begin{array}{r}
x^{\prime}=x \cos \omega t+y \sin \omega t \\
y^{\prime}=-x \sin \omega t+y \cos \omega t
\end{array}
$$

here $\omega$ represents the angular velocity of rotation. The corresponding derivatives then transform in the following way:

$$
\begin{aligned}
\frac{\partial}{\partial x} & =\cos \omega t \frac{\partial}{\partial x^{\prime}}-\sin \omega t \frac{\partial}{\partial y^{\prime}} \\
\frac{\partial}{\partial y} & =\sin \omega t \frac{\partial}{\partial x^{\prime}}+\cos \omega t \frac{\partial}{\partial y^{\prime}}
\end{aligned}
$$

Under this transformation the energy density of the static Hamiltonian in Equation (1) transforms in a simple way:

$$
\left[\left(\frac{\partial \mathbf{n}}{\partial x}\right)^{2}+\left(\frac{\partial \mathbf{n}}{\partial y}\right)^{2}\right]=\left[\left(\frac{\partial \mathbf{n}}{\partial x^{\prime}}\right)^{2}+\left(\frac{\partial \mathbf{n}}{\partial y^{\prime}}\right)^{2}\right]
$$

Because of the rotation we have to consider the full dynamical hamiltonian density:

$$
\frac{1}{c^{2}}\left(\frac{d \mathbf{n}}{d t}\right)^{2}+J_{0}\left[\left(\frac{\partial \mathbf{n}}{\partial x}\right)^{2}+\left(\frac{\partial \mathbf{n}}{\partial y}\right)^{2}\right]
$$

In the context of an anti-ferromagnet $[5,6]$ e.g. $c$ is the spin velocity and $J_{0}$ is the spin interaction constant. We note that the implicit dependence of $\mathbf{n}$ on time is through $x^{\prime}(t)$ and $y^{\prime}(t)$. Now let us calculate the kinetic term in the rotating coordinate system:

$$
\begin{aligned}
\frac{1}{c^{2}}\left(\frac{d \mathbf{n}}{d t}\right)^{2}= & \frac{1}{c^{2}}\left[\frac{\partial \mathbf{n}}{\partial x^{\prime}} \frac{d x^{\prime}}{d t}+\frac{\partial \mathbf{n}}{\partial y^{\prime}} \frac{d y^{\prime}}{d t}\right]^{2} \\
& =\frac{\omega^{2}}{c^{2}}\left[y^{\prime} \frac{\partial \mathbf{n}}{\partial x^{\prime}}-x^{\prime} \frac{\partial \mathbf{n}}{\partial y^{\prime}}\right]^{2}
\end{aligned}
$$


The total static Hamiltonian in the rotating coordinate system now reads [7]:

$$
\begin{aligned}
& H^{\prime}=\iint \frac{\omega^{2}}{c^{2}}\left[y^{\prime} \frac{\partial \mathbf{n}}{\partial x^{\prime}}-x^{\prime} \frac{\partial \mathbf{n}}{\partial y^{\prime}}\right]^{2} d x^{\prime} d y^{\prime} \\
& +\iint J_{0}\left[\left(\frac{\partial \mathbf{n}}{\partial x^{\prime}}\right)^{2}+\left(\frac{\partial \mathbf{n}}{\partial y^{\prime}}\right)^{2}\right] d x^{\prime} d y^{\prime}
\end{aligned}
$$

Now it is obvious that the Hamiltonian $H^{\prime}$ is not invariant under stretching $x^{\prime} \rightarrow \lambda x^{\prime}$ and $y^{\prime} \rightarrow \lambda y^{\prime}$ so possible skyrmions will not collapse in one point as in the case of the non rotating disk. Let us note another interesting feature of the Hamiltonian $H^{\prime}$ : this is the appearance of the Gaussian curvature $K=-3 \frac{\omega^{2}}{c^{2}}$ for small $\frac{\omega}{c}<<1$ Indeed the line element in the non rotating plane is $d s^{2}=d x^{2}+d y^{2}$ and obviously the curvature $K=0$. In the rotating disk the line element is $d s^{2}=d r^{2}+h(r)^{2} d \phi^{2}$ where $h(r)=\frac{r}{1-\omega^{2} r^{2} / c^{2}}$. The curvature is $K=-\frac{1}{h} \frac{\partial^{2} h}{\partial r^{2}} \neq 0[8]$. The Hamiltonian $H^{\prime}$ reads:

$$
\begin{aligned}
& H^{\prime}=\iint \frac{1}{3}|K|\left[y^{\prime} \frac{\partial \mathbf{n}}{\partial x^{\prime}}-x^{\prime} \frac{\partial \mathbf{n}}{\partial y^{\prime}}\right]^{2} d x^{\prime} d y \\
& +\iint J_{0}\left[\left(\frac{\partial \mathbf{n}}{\partial x^{\prime}}\right)^{2}+\left(\frac{\partial \mathbf{n}}{\partial y^{\prime}}\right)^{2}\right] d x^{\prime} d y^{\prime}
\end{aligned}
$$

The rotation introduces curvature and hence length in the problem which stabilises possible skyrmions. In order to elucidate the role of the Gaussian curvature we are going to rewrite the Hamiltonian $H^{\prime}$ in polar coordinates:

$$
x^{\prime}=r \cos \theta \quad y^{\prime}=r \sin \theta
$$

Now we will use the fact that the vector $\mathbf{n}$ is normalised and will write it in the following form: $\mathbf{n}=(\sin \theta \cos \Phi, \sin \theta \sin \Phi, \cos \theta)$. We will consider only cylindrically symmetric configuration i.e. $\Phi=\phi$ The new Hamiltonian density now reads:

$$
H^{\prime}=\iint\left[J_{0}\left[\left(\frac{d \theta}{d r}\right)^{2}+\frac{\sin ^{2} \theta}{r^{2}}\right]+\frac{1}{3}|K|\left[\frac{\partial \mathbf{n}}{\partial \phi}\right]^{2}\right] r d r d \theta
$$

where $\mathbf{L}=\sqrt{\frac{1}{3}|K|} \frac{\partial \mathbf{n}}{\partial \phi}$ plays the role of an "angular momentum" for the vector field [7]. Now because $\left(\frac{\partial \mathbf{n}}{\partial \phi}\right)^{2}=\sin ^{2} \theta$ the Hamiltonian reads:

$$
H^{\prime}=\iint\left[J_{0}\left[\left(\frac{d \theta}{d r}\right)^{2}+\frac{\sin ^{2} \theta}{r^{2}}\right]+\frac{1}{3}|K| \sin ^{2} \theta\right] r d r d \theta
$$

The presence in the Hamiltonian of the "angular momentum" of the field $\mathbf{L}$ prevents the Hamiltonian of being scale invariant as it is in the case of the non rotating disk. The Euler-Lagrange equation related to this Hamiltonian reads:

$$
J_{0} \frac{d \theta}{d r}+J_{0} r \frac{d^{2} \theta}{d r^{2}}=\left(\frac{J_{0}}{r}+\frac{|K| r}{3}\right) \sin \theta
$$


Here we introduce the following change of variables: $\bar{r}=\ln \frac{r}{r_{0}}$ or $r=r_{0} e^{\bar{r}} . r_{0}$ is a very small radius around the origine of the coordinate system where the system is not well defined [9]. In the new variable $\bar{r}$ the Euler-Lagrange equation becomes a sine-Gordon equation with variable coefficients.

$$
\frac{d^{2} \theta}{d \bar{r}^{2}}=\left(1+\frac{|K| r_{0}^{2} e^{2 \bar{r}}}{3 J_{0}}\right) \sin \theta
$$

This equation has to be solved numerically. For flat disk (non rotating) where the curvature is zero $|K|=0$, we recover the radial sine-Gordon equation.

\section{Conclusion}

The 2D skyrmions on the plane are metastable because they can be scaled to a point. Usually these skyrmions are stabilised by introducing length into the problem: e.g. the cylinder, or the plane with a hole cut in it, or the introduction of an external magnetic field which introduces length. We have shown here that even without manipulating the underlying manifold (the plane) by cutting, or bending, we can stll introduce length into the problem via rotation of the plane. The rotation introduces curvature and hence length.

\section{Conflicts of Interest}

The author declares no conflicts of interest regarding the publication of this paper.

\section{References}

[1] Belavin, A.A. and Polyakov, A.M. (1975) JETP Letters, 22, 245.

[2] Dandoloff, R. and Saxena, A. (1977) Zeitschrift für Physik B, 104, 1405.

[3] Villain-Guillot, S., Dandoloff, R., Saxena, A. and Bishop, A.R. (1995) Physical Review B, 52, 6712. https://doi.org/10.1103/PhysRevB.52.6712

[4] Shankar, R. (1977) Journal de Physique, 38, 1405-1412. https://doi.org/10.1051/jphys:0197700380110140500

[5] Haldane, F.D.M. (1988) Physical Review Letters, 61, 1029. https://doi.org/10.1103/PhysRevLett.61.1029

[6] Baskaran, G. (2011) Possibility of Skyrmion Superconductivity in Doped Antiferromagnet $\mathrm{K}_{2} \mathrm{Fe}_{4} \mathrm{Se}_{5}$. arXiv:1108.3562v1

[7] Derrick, G.H. (1964) Journal of Mathematical Physics, 5, 1252. https://doi.org/10.1063/1.1704233

[8] Atanasov, V. and Dandoloff, R. (2015) Physica Scripta, 90, Article ID: 065001. https://doi.org/10.1088/0031-8949/90/6/065001

[9] Saxena, A. and Dandoloff, R. (2002) Physical Review B, 66, Article ID: 104414. 